\title{
Erratum: A selective inhibitor of the immunoproteasome subunit LMP7 blocks cytokine production and attenuates progression of experimental arthritis
}

Tony Muchamuel, Michael Basler, Monette A Aujay, Erika Suzuki, Khalid W Kalim, Christoph Lauer, Catherine Sylvain, Eileen R Ring, Jamie Shields, Jing Jiang, Peter Shwonek, Francesco Parlati, Susan D Demo, Mark K Bennett, Christopher J Kirk \& Marcus Groettrup Nat. Med. 15, 781-787 (2009); published online 14 June 2009; corrected after print 5 November 2009

In the version of this article initially published, the structure of PR-957 in Figure 1a was incorrect. An -NH group was missing. The error has been corrected in the HTML and PDF versions of the article.

\section{Corrigendum: Baseline Ad5 serostatus does not predict Ad5 HIV vaccine- induced expansion of adenovirus-specific CD4 ${ }^{+} \mathrm{T}$ cells}

Natalie A Hutnick, Diane G Carnathan, Sheri A Dubey, George Makedonas, Kara S Cox, Lisa Kierstead, Sarah J Ratcliffe, Michael N Robertson, Danilo R Casimiro, Hildegund C J Ertl \& Michael R Betts

Nat. Med. 15, 876-878 (2009); published online 20 July 2009; corrected after print 5 November 2009

In the version of this article initially published, George Makedonas was omitted from the author list. The error has been corrected in the HTML and PDF versions of the article. 